\title{
Comique et autobiographique dans les contes de Sade
}

\section{Francesco Fiorentino}

\section{(2) OpenEdition}

\section{Journals}

\section{Édition électronique}

URL : http://journals.openedition.org/rief/1055

DOI : 10.4000/rief.1055

ISSN : 2240-7456

\section{Éditeur}

Seminario di filologia francese

\section{Référence électronique}

Francesco Fiorentino, "Comique et autobiographique dans les contes de Sade », Revue italienne d'études françaises [En ligne], 5 | 2015, mis en ligne le 15 décembre 2015, consulté le 19 avril 2019 URL : http://journals.openedition.org/rief/1055 ; DOI : 10.4000/rief.1055

Ce document a été généré automatiquement le 19 avril 2019

\section{(c) (i) (9)}

Les contenus de la RIEF sont mis à disposition selon les termes de la Licence Creative Commons Attribution - Pas d'Utilisation Commerciale - Pas de Modification 4.0 International. 


\title{
Comique et autobiographique dans les contes de Sade
}

\author{
Francesco Fiorentino
}

1 Parmi les œuvres de Sade, ses contes ont été le dernier ouvrage à avoir été découvert. Si Les Crimes de l'amour furent publiés chez Massé en l'an VIII (1800), pour les autres contes il faut attendre les deux éditions de 1926 et de 1927 de Maurice Heine.

2 Les lecteurs non spécialistes les ont ignorés encore plus longtemps. Les Crimes de l'amour et les Historiettes, contes et fabliaux n'ont été disponibles dans une collection grand public qu'en 1965 et en 1971 grâce aux éditions 10/18 qui ont repris la version de 1958 par Gilbert Lely. L'intérêt des lecteurs pour ces contes n'a cependant grandi qu'à partir de 1987 grâce à l'édition de Michel Delon des Crimes de l'amour et à celle d'Annie Le Brun et Jean-Jacques Pauvert dans le volume X des Euvres complètes de Sade. En 2014, année anniversaire de sa mort, deux éditions commentées de ses contes ont enfin paru: Contes libertins par Stéphanie Genand chez Garnier-Flammarion qui a recueilli certains de ces contes sur la base de leur sujet et Contes étranges par Michel Delon chez Folio classique qui a réuni tous les contes non publiés par Sade dans Les Crimes de l'amour. Ce n'est qu'à propos de ce dernier recueil que je vais vous proposer quelques observations.

3 Cette longue négligence des contes sadiens a peut-être eu aussi une motivation relative au champ littéraire: jusqu'aux années 1970 la figure de Sade était au centre du débat philosophique plutôt que littéraire et l'attention des critiques était concentrée surtout sur ses ouvrages extrêmes avec un contenu idéologique très transgressif, à commencer par Les cent vingt journées de Sodome. L'image de Sade nouvelliste, sans la contredire, était quand même étrangère à celle du théoricien précoce de la catastrophe moderne dont à l'époque on voulait l'accréditer. Admettre l'importance des contes (et des œuvres théâtrales) comportait de reconnaitre un statut exclusivement littéraire (pas de cas clinique, ni de cas philosophique non plus) à l'œuvre de Sade ${ }^{1}$ : statut qui d'ailleurs correspondait à l'ambition de l'auteur qui aspirait à la réputation d'homme de lettres comme justement les contes en témoignent. Il avait en effet attribué une importance considérable à leur composition : ceux qui nous sont parvenus faisaient partie d'un projet 
bien plus vaste qui en 1788 contemplait quatre volumes « de nouvelles sérieuses et de contes gais », projet encore reconsidéré en 1803. Comme Michel Delon l'a observé, la nouvelle a retenu l'attention de l'écrivain tout au long de sa carrière littéraire ${ }^{2}$.

4 Non seulement les Crimes de l'amour, mais tout le reste des contes aussi, bien qu'ils n'aient pas été publiés de son vivant, partagent le même registre stylistique et moral que celui des textes publiés par l'auteur avec sa signature (comme le roman Aline et Valcourt) : à propos de ces ouvrages Jean Roussel a parlé d'un Sade diurne qui aspire à une réputation et qu'il oppose à un Sade nocturne ${ }^{3}$, tandis que Michel Delon a désigné le même contraste comme celui entre un Sade ésotérique et un Sade exotérique ${ }^{4}$. Toujours Michel Delon distingue le signifié idéologique de ces contes ésotériques par rapport aux ouvrages exotériques:

Une morale se dégage de l'ensemble de ces contes, une fois écartés Les Crimes de l'amour et leur grandeur tragique, abrupte. Ils évitent la noirceur absolue et préfèrent les accommodements [...] Le rire remplace le sang [...] L'indulgence remédie aux faiblesses, la tolérance prône les arrangements. ${ }^{5}$

Delon néanmoins observe que, bien qu'apparemment conciliants et débonnaires, ces contes entretiennent eux aussi des liens avec l'autre monde de la démesure et de la transgression :

Il reste d'inquiétantes fêlures du réel : la violence emporte parfois les individus audelà de toute limite, le désir submerge d'une jouissance excessive, tout échange, tout compromis deviennent illusoires dans cette quête de l'impossible. ${ }^{6}$

Les contes en effet ont été créés au cours de la même période et dans le même atelier (la prison) des Cent vingt journées de Sodome, le livre «maudit» par excellence. Et cette proximité, comme Delon lui-même le suggère, est visible en particulier dans un texte comme Émilie de Tourville.

Ce conte présente des analogies évidentes avec le roman Justine ou les Malheurs de la vertu que Sade écrivait à la même époque, comme développement d'une nouvelle, Les Infortunes de la vertu. La comparaison entre ces deux ouvrages se prête donc tout particulièrement à illustrer les constantes et les variantes de ces deux registres narratifs présents dans la production sadienne, dont Delon et Roussel parlent. Comme Justine débute en tant qu' exemplum qui répond à la question "philosophique " sur l'existence de la Providence, Émilie de Tourville aussi part d'une interrogation morale dont l'ouvrage se présente comme une illustration : y a-t-il une limite à ce qu'on peut faire au nom de l'honneur? Et plus en général, faut-il une proportionnalité entre la faute et la punition, come le soutenait Beccaria? Cette question est cruellement actuelle pour le prisonnier qui déclare n'avoir été enfermé qu'à cause d'une bagatelle.

Comme la première Justine, Émilie aussi raconte sa propre histoire : son destinataire est le comte de Luxeuil qui l'a sauvée après l'avoir trouvée dans un bois saignée à blanc. Tombée amoureuse d'un jeune homme, elle avait accepté de le rencontrer chez une femme complaisante. Cette femme se révèle une entremetteuse sans scrupules et, profitant de l'absence de son amoureux, la conduit par la ruse chez un vieux libertin. Renseigné, l'amoureux non seulement répudie Émilie mais la dénonce aussi à ses frères qui la détestent et profitent de sa faute. Sous le prétexte de venger leur honneur, ils l'enferment dans une maison de campagne gardée par deux serviteurs qui doivent la saigner tous les quatre jours aux deux bras.

9 Dans Justine, le libertin Gernande inflige le même martyre à sa femme. C'est surtout le même mécanisme narratif du conte qui se répète dans le roman. Justine, dès qu'elle a 
échappé à des sévices, se plaint de son sort avec un interlocuteur nouveau qui, loin de la secourir, profite à son tour d'elle. À la fin de la narration d'Émilie donc, le lecteur sadien s'attend à ce que la jeune femme doive subir une attaque nouvelle. Au contraire, Luxeuil se révèle un protecteur généreux et, en découvrant qu'il est le père de l'amoureux d'Émilie, il obtient que son fils se repente et se marie avec elle. L'histoire d'Émilie est aussi cruelle que l'histoire de Justine : toutes les deux à cause de leur ingénuité sont des victimes prédestinées à endurer les vexations de leurs persécuteurs; toutes les deux témoignent de l'injustice terrestre et céleste; dans les deux narrations, des passions excessives se déchaînent avec des conséquences funestes. Mais, tandis que le roman ne prévoit pas une catharsis, le conte redresse l'histoire vers une fin heureuse. Ce happy end cependant ne se présente pas comme une solution mièvre: il se réalise grâce à une transgression des règles morales et littéraires en vigueur à l'époque. En effet, contrairement à Justine, Émilie n'est pas innocente car elle est coupable d'avoir étourdiment compromis l'honneur de sa famille; à son tour son amoureux est responsable de la bassesse de l'avoir dénoncée à ses frères. Mais ces fautes n'empêchent pas qu'à la fin le bonheur leur soit réservé. Cette justice supérieure, capable de comprendre les raisons du coupable, qui n'est pas vindicative - celle que le prisonnier invoque inutilement pour lui-même - se réalise grâce à M. de Luxeuil. Son personnage, qui paraît le contraire de celui du libertin, partage quand même une prérogative avec lui. Luxeuil aussi se révèle être le maître de la vie de l'autre, dans son cas en exerçant la justice : le droit réservé au seigneur féodal que l'État moderne, selon Sade, a usurpé.

10 Le thème de la justice et surtout de la polémique contre ceux qui l'administrent est une sorte de fil rouge qui traverse plusieurs de ces contes. En 1772 Sade est condamné à mort par contumace et exécuté en effigie à cause de l'orgie avec quatre prostitués (durant laquelle il pratique la sodomie, la flagellation et emploie la cantharide), en 1773 il arrive à s'évader. Mais en 1777 il est capturé et emprisonné : pendant douze ans sa vie sera celle d'un prisonnier, d'abord à Vincennes, puis à la Bastille, enfin à Charenton. Les contes donnent volontiers voix à sa protestation et à son désir de vengeance contre les juges. En particulier le plus long de ces contes, Le président mystifié, prétend être une véritable reddition des comptes avec la catégorie détestée.

11 Le président du tribunal d'Aix (la ville où Sade a été condamné), vieux et prétentieux, se marie avec Mlle de Téroze, une fille aristocratique, jeune et belle. La sœur de Mlle de Téroze, son beau frère le marquis d'olincourt et son jeune amant, pour punir et convaincre le détestable mari à partir, organisent quatorze ${ }^{7}$ "mystifications" (les mystifications étant des tromperies où tous sont complices pour duper quelqu'un). Le ridicule président est purgé, jeté dans une fosse à fumier, mis au lit avec une mégère noire et avec un âne, convaincu d'être fou, plongé dans l'eau froide, brûlé sur des charbons ardents, battu par des présumés revenants, cocufié sous ses yeux sur un ballon aérostatique, séduit par une femme complaisante pour pouvoir être surpris en flagrant adultère. À la fin, il renonce aux noces et revient sage dans sa province.

Bien qu'elles inspirent des tirades éloquentes du marquis d'olincourt et des allusions explicites à ses vicissitudes judiciaires, dans ce conte la rancune de Sade envers les juges, sa douleur et son indignation s'expriment pour une fois dans un registre comique. Le conte en effet se présente comme une sorte de réécriture narrative d'une farce. Pour la forme : parce qu'après la courte exposition, il est organisé comme une pièce, en scènes et dialogues; et pour le contenu, comme l'observe le marquis d'olincourt lui-même : « Vous le voyez, mon ami, ce monde-ci n'est qu'une farce $»^{8}$. C'est surtout le président Fontanis 
qui est farcesque : il est dégradé par une comparaison continuelle avec les animaux et par l'accent posé sur la monstruosité de sa figure, à partir de sa bouche sans dents qui ressemble à une latrine9. Les épreuves hyperboliques, auxquelles il est soumis, sont absorbées par son corps qui est toujours comiquement (et pas vraisemblablement) prêt pour une nouvelle torture. Sa vanité, dans un autre domaine, est la même que celle des capitans fanfarons et des médecins de la commedia dell'arte, sa couardise est celle des valets poltrons. Le conte en effet me semble la reprise assez ponctuelle de Monsieur de Pourceaugnac (1670) de Molière. Sbrigani et Nerine soumettent Pourceaugnac, venu à Paris pour épouser Julie, à toute une série de mystifications : on le fait passer pour fou, pour un commerçant ruiné, pour bigame; travesti en femme, il doit subir les avances de deux soldats et il est menacé d'arrestation. Enfin le pauvre Pourceaugnac s'enfuit de Paris et Julie peut se marier avec son amoureux.

13 L'intrigue est évidemment la même: Sade qui dans son théâtre ne se tourne pas vers Molière, le reprend dans son conte. Et pas seulement pour Pourceaugnac. Le président regrette de s'être marié par ambition : « tu l'as voulu, pauvre président, tu l'as voulu, mon ami, t'y voilà, un mariage de Paris t'a tenté, tu vois ce qu'il en résulte», se dit-il ${ }^{10}$. Cette réplique rappelle la plainte célèbre de George Dandin : «Vous l'avez voulu, vous l'avez voulu, George Dandin ». Comme Dandin, le président a voulu une femme de noblesse supérieure et, dans la perspective plus qu'aristocratique féodale de Sade, cette prétention est une faute très grave. Et c'est toujours de Molière, comme on l'a signalé, que Sade s'inspire pour ses tirades contre les juges qui reprennent celles contre les médecins (mais c'est sûr qu'il connaissait aussi Les Plaideurs de Racine). Dans deux passages les deux professions sont assimilées: dans une répartie de d'olincourt : «je n'ai pas à l'exemple des enfants de Thémis et d'Esculape la manie sanguinaire... ${ }^{11}$ et dans une répartie de Fontanis: "...nous voulons comme les médecins tuer indifféremment qui bon nous semble, sans que le défunt ait jamais rien à nous dire ${ }^{12}$. Comme le montre cette dernière répartie, l'arrogance déraisonnable à la manière d'Ubu de Fontanis peut se traduire dans une sorte de candeur cynique qui ne manque pas d'esprit. Encore : « je suis un peu comme César, le courage est très journalier chez moi... $»^{13}$. Ainsi l'abjection comique du personnage est parfois en partie rachetée par un trait qu'avec Freud du Mot d'esprit et ses rapports avec l'inconscient nous pouvons appeler wizzig.

14 Ce caractère ambigu du comique risque de transformer le président persécuté par tout le monde, qui résiste à toute vexation, en une victime capable d'inspirer de la pitié et une certaine sympathie chez le lecteur. Le persécuteur arrogant pourrait se transformer en " un pauvre diable », comme le définit à juste titre le narrateur lui-même ${ }^{14}$ (et il est aussi désigné comme "le pauvre Provincial $»^{15}$ ). Cette ambiguïté est inscrite dans le mécanisme même de la Mystification qui voit l'association de tout le monde contre une personne qui en fait les frais. La mystification revient dans d'autres contes et représente évidemment une des obsessions du prisonnier qui se sent persécuté par une combine générale. Dans $L a$ Marquise de Télème, conte inachevé, la pauvre marquise est victime de libertins qui profitent de ses ennuis judiciaires. Dans Les Filous, qui avait reçu comme titre précédent La Provinciale mystifiée, une jeune fille, gâtée par son père bourgeois provincial, prétend aller toute seule à Paris pour rencontrer des parents qu'elle ne connaît pas. Une bande de canailles fait semblant d'être ces parents, l'enivre, lui vole son argent et ses vêtements, la viole et l'abandonne le lendemain devant l'église Saint-Roch. La source de ce conte, comme de plusieurs autres contes de Sade, a été indiquée par Michel Delon dans Les Lettres historiques et galantes de deux dames de condition de Mme Du Noyer (1707-1717). Mais, 
par rapport à la source qui accuse la jeune fille pour son comportement téméraire, Sade souligne au contraire son ingénuité pathétique. L'alliance de tout le monde contre une personne seule le touche, en alimentant en même temps sa paranoïa de persécuté comme son délire de persécuteur. Comme l'a observé Michel Delon ${ }^{16}$, les souffrances du président en présence de tout le monde (donc en partageant la perspective des persécuteurs) rappellent - dans un autre registre - celles de Justine (souffrances au contraire regardées du côté de la victime). Les deux positions de victime isolée et de persécuteur, comme l'a remarqué Jean Paulhan ${ }^{17}$, sont liées d'une façon indissoluble par le désir. Ces obsessions constituent probablement un des mobiles psychologiques fondamentaux de toute l'œuvre de Sade qui présente toujours des situations qui comportent l'identification du lecteur avec la victime ou avec les libertins qui en jouissent. Ou les deux ensemble.

La mystification dont il est victime risque donc d'attirer quelque sympathie envers le président. Le narrateur aussi confirme ce risque et il essaye de remédier à la fin du conte avec une répartie du marquis qui soulève la question : «J'ai quelquefois un peu de regret d'avoir autant maltraité ce vilain homme». D'Olincourt peut ainsi se justifier en observant que, grâce à ce mauvais traitement, sa belle-sœur a pu se marier avec son amant et surtout parce que le président vexé n'est qu' « un drôle inutile à la société, foncièrement ennemi de l'Etat, perturbateur du repos publique, fourreau d'une famille honnête et respectueuse, diffamateur insigne d'un gentilhomme que j'estime... ». Escusatio non petita.... Le passage du tragique au comique crée une distance explicite par rapport au personnage, mais, comme nous l'avons vu, n'élimine pas toute possibilité d'identification avec elle.

Le désir d'un dédommagement envers les juges balance dans ces contes une autre inspiration, moins virulente, celle qui se rattache à la tradition de la nouvelle de Boccace et de la Renaissance (le projet de Sade en 1803 était de publier un Boccace français). Mais une veine cruelle peut s'insinuer aussi dans ce registre plus gai. Je pense à La Femme vengée, où deux nobles mariés ont chacun un amant, dans l'entente parfaite de leur mariage. Mais le sire de Longueville ne peut pas accepter d'être cocu et décide de faire tuer Colas, en le prenant la nuit quand il rejoint la chambre de sa femme. Il sera mis dans un sac et jeté dans l'eau. La femme, qui arrive à savoir le projet du mari, en profitant de l'obscurité substitue l'amante de son mari à son amant: elle sera donc tuée à sa place. Frappé par la mystérieuse substitution, le sire de Longueville décide de devenir fidèle et sa femme accepte de sacrifier son Colas. Le fait que l'entente conjugale nouvelle coûte la vie d'une jeune fille (Sade semble prévoir le sadisme de Musset de On ne badine pas avec l'amour) ne trouble pas le happy end. Comme dans Le Mari corrigé l'expédient de la femme qui se donne à son amant devant son mari pour le punir de se limiter à la fesser n'empêche pas la réconciliation conjugale. En effet la fin heureuse et cynique rentre dans le code de ce type de nouvelle. Le fait que la situation initiale de la nouvelle soit semblable à la situation finale me semble plus transgressif : les mariés vivaient heureux chacun avec son amant, comme à la fin quand ils sont fidèles. Le bonheur fait abstraction de la morale comme des règles sociales. Faire abstraction de la morale peut être en effet encore plus scandaleux que la renverser. 


\section{NOTES}

1. Cf. S. Genand, Présentation à Sade, Contes libertins, Paris, Garnier-Flammarion, 2014, p. 9.

2. M. Delon, Préface à Sade, Contes étranges, Paris, Gallimard, « Folio classique », 2014, p. 12-13.

3. J. Roussel, «Les Crimes de l'amour : le sadisme dans l'écriture décente », dans Studi in onore di Mario Matucci, Pisa, Pacini, 1993, p. 91.

4. M. Delon, «De Thérèse philosophe à La Philosophie dans le boudoir ", dans Romanistische Zeitschrift für Literaturgeschichte, vol 16, n.1-2, p. 76.

5. M. Delon, Préface à Sade, Contes étranges, cit., p. 24

6. Ibid., p. 25.

7. Mais Sade, qui les compte dans une annotation du manuscrit, se trompe : il y en a quinze.

8. Le Président mystifié, dans Contes étranges cit, p. 191-92.

9. Ibid., 152.

10. Ibid., 197.

11. Ibid., 178.

12. Ibid., 194.

13. Ibid., 196.

14. Ibid., 182.

15. Ibid., 168.

16. Ibid., 218.

17. J. Paulhan, La Douteuse Justine ou les revanches de la pudeur, préface aux Infortunes de la vertu, Paris, Pauvert, 1946.

\section{INDEX}

Mots-clés : Sade (Donatien Alphonse François marquis de), conte au XVIIIe siècle, justice (thème de la), comique (caractère ambigu du), tradition de la nouvelle 